\title{
SLIDING MODE METHODS FOR FAULT DETECTION AND FAULT TOLERANT CONTROL WITH APPLICATION TO AEROSPACE SYSTEMS
}

\author{
Christopher EDWARDS *, Halim ALWI *, Chee Pin TAN ** \\ ${ }^{*}$ Department of Engineering \\ University of Leicester, University Road, Leicester, LE1 7RH, UK \\ e-mail: \{ce14, ha18\}@le.ac.uk \\ ${ }^{* *}$ School of Engineering, Sunway Campus \\ Monash University, Jalan Lagoon Selatan, 46150 Sunway, Selangor, Malaysia \\ e-mail: tan.chee.pin@eng.monash.edu.my
}

\begin{abstract}
Sliding mode methods have been historically studied because of their strong robustness properties with regard to a certain class of uncertainty, achieved by employing nonlinear control/injection signals to force the system trajectories to attain in finite time a motion along a surface in the state-space. This paper will consider how these ideas can be exploited for fault detection (specifically fault signal estimation) and subsequently fault tolerant control. It will also describe applications of these ideas to aerospace systems, including piloted flight simulator results associated with the GARTEUR AG16 Action Group on Fault Tolerant Control. The results demonstrate a successful real-time implementation of the proposed fault tolerant control scheme on a motion flight simulator configured to represent the post-failure EL-AL aircraft.
\end{abstract}

Keywords: sliding modes, fault detection, fault tolerant control, control allocation.

\section{Introduction}

The fundamental purpose of a Fault Detection and Isolation (FDI) scheme is to generate an alarm when a fault occurs and to pin-point the source (Patton et al., 1989). Fault Tolerant Control (FTC) systems seek to provide, at worst, a degraded level of performance (compared to the fault free situation) in the event of a fault or failure developing in the system. Most existing FDI schemes in the literature are concerned with the design of the so-called residuals. These residual signals are used as 'alarms' to indicate the occurrence of a fault and, if properly designed, give information from which the source of the fault may be identified.

In analytic redundancy approaches, the residuals are (usually dynamic) weightings of the difference between the measured plant output and the output of a model of the system. Many fault detection methods are observer based; the observer will usually be designed from a model which will inevitably not be a perfect representation of the real system. In terms of the observer design, the plant/model mismatch will usually be encapsulated as uncertainty. The design procedure for the FDI scheme must then seek to mitigate the effect of the uncertainty on the residuals in an effort to minimize false alarms and missed faults when the scheme is implemented on a real system (Chen and Patton, 1999).

In the last decade the use of sliding mode observers for FDI has been explored. The novelty of the approach lies in the ability of sliding mode observers to reconstruct unmeasurable signals within a process by appropriate scaling and filtering of the so-called 'equivalent output error injection' (Edwards et al., 2000). This is a unique property of sliding mode observers, which emanates from the fact that the introduction of a sliding motion forces the outputs of the observer to perfectly track the plant measurements (Edwards et al., 2000). Reconstruction approaches attempt to capture both the magnitude and 'shape' of the faults, which can be advantageous.

The fact that even in the presence of faults the output of the sliding mode observer still perfectly follows the plant output means that residuals formulated in the usual way, i.e., as functions of the output estimation error, would always be zero. Instead, the effect of the faults is seen through the fact that the equivalent output error injection term must compensate for the fault in order to maintain sliding. The work of Edwards et al. (2000) relies on the 
assumption that the transfer function matrix relating the faults to the measurement signals has relative degree one minimum phase properties. Robustness to uncertainty in the modelling process is vital. Edwards et al. (2000) as well as Edwards and Spurgeon (2000) used a sliding mode observer to reconstruct faults, in which there was no explicit consideration of the disturbances or uncertainty. Tan and Edwards (2003) built on the work of Edwards and Spurgeon (2000) as well as Edwards et al. (2000) and presented a design algorithm for the observer, using Linear Matrix Inequalities (LMIs) (Boyd et al., 1994), such that the $\mathcal{L}_{2}$ gain from the disturbances to the fault reconstruction is minimized. Subsequent work has sought to develop schemes which relax the conditions imposed by Edwards et al. (2000).

FDI schemes often represent only a subcomponent of the overall control architecture. In safety critical systems, there is an inherent requirement that, overall, some level of possibly degraded performance must be maintained even in the event of serious faults or failures occurring within the system. The ability to deal with situations in which faults and failures occur originally coined the term 'self repairing control', although now this is more commonly referred to by the moniker 'fault tolerant control'.

Generally speaking, fault tolerant control schemes are classified as either passive or active (Blanke et al., 2006). Passive schemes operate independently of any fault information and basically exploit the robustness of the underlying control paradigm (Blanke et al., 2006; Patton, 1997). Such schemes are usually less complex, but in order to cope with 'worst case' fault effects they are conservative. In this situation, nominal performance must often be sacrificed to achieve fault tolerance (Banda, 1999). Active fault tolerant controllers react to the occurrence of faults, typically by using information from a fault detection and isolation scheme, and invoke some form of reconfiguration. This represents a more flexible architecture.

In some situations the faults can be accommodated, i.e., a new controller can be found (at least theoretically) to recover an acceptable level of performance (Blanke et al., 2006). Reconfiguration is usually necessary in the event of severe faults such as total failures in actuators/sensors. For example, if a sensor or actuator fails totally, no adaptation within that feedback loop can recover performance without modification to the choice of actuators and sensors coupled via the controller (i.e., reconfiguration). Furthermore, often the reference trajectory needs to be reconfigured to acknowledge the loss of performance as a result of faults and failures (Theilliol et al., 2008).

Historically, sliding mode concepts have been the focus of research because of their robustness to the so-called matched uncertainty (Utkin, 1992). The possibilities of exploiting the inherent robustness properties of sliding modes for fault tolerance has previously been explored for aerospace applications (Hess and Wells, 2003; Shtessel et al., 2002). In fact, the work of Hess and Wells (2003) argued that sliding mode control has the potential to become an alternative to reconfigurable control.

This paper will describe how sliding mode ideas can be exploited for fault detection (specifically fault signal estimation) and subsequently fault tolerant control. It will also describe applications of these ideas to aerospace systems and describe piloted flight simulator results associated with the GARTEUR AG16 action group on fault tolerant control. The results demonstrate a successful realtime implementation of the proposed fault tolerant control scheme on a motion flight simulator configured to represent the EL-AL aircraft associated with the Bijlmermeer incident (Edwards et al., 2010).

\section{First order sliding mode observers}

Historically, sliding mode ideas emerged from the former USSR in the 1950s (Utkin, 1992). Usually, these ideas are discussed for control system design, in which case the control law is designed to drive the states onto and forces them to remain on a predetermined surface in the state space. The motion while constrained to the surface is termed the sliding motion. There are two advantages of this approach:

- the sliding motion is of lower order than the original system;

- sliding mode systems exhibit insensitivity properties to the so-called matched uncertainty (Draženović, 1969)

The latter property has fuelled research in the area of sliding modes (and this robustness can be exploited for fault tolerant control). In this section, sliding modes will be considered from the perspective of observer design.

As an example consider the equations of motion for a pendulum

$$
\ddot{\phi}(t)=-\sin (\phi(t))
$$

written as

$$
\dot{x}(t)=\left[\begin{array}{ll}
0 & 1 \\
0 & 0
\end{array}\right] x(t)+\left[\begin{array}{l}
0 \\
1
\end{array}\right] \xi(t, x),
$$

where $x_{1}=\phi, x_{2}=\dot{\phi}$ and $\xi(t, x)=-\sin (\phi)$. Artificially choose $y(t)=C x(t)$, where

$$
C=\left[\begin{array}{ll}
1 & 1
\end{array}\right] \text {. }
$$

The aim is to simultaneously estimate both $x(t)$ and $\xi(t, x)$ from $y(t)$ and $u(t)$. A sliding mode observer is given by

$$
\dot{z}(t)=\left[\begin{array}{ll}
0 & 1 \\
0 & 0
\end{array}\right] z(t)-\left[\begin{array}{l}
1 \\
1
\end{array}\right] e_{y}(t)-\left[\begin{array}{l}
0 \\
1
\end{array}\right] \underbrace{2 \operatorname{sign}\left(e_{y}\right)}_{\nu},
$$




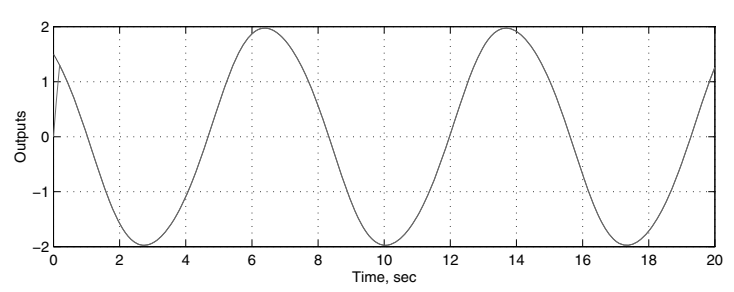

Fig. 1. Comparison of the outputs from the plant and the observer.

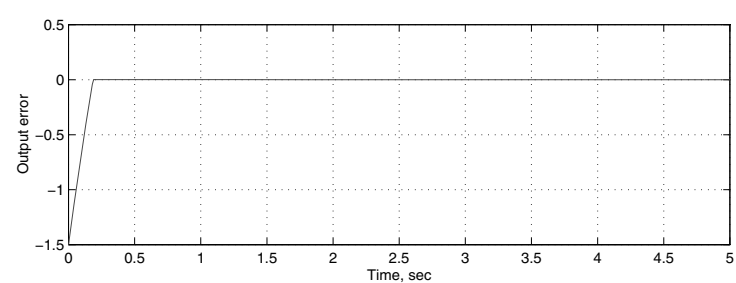

Fig. 2. Output estimation error.

where $e_{y}(t)=C z(t)-y(t)$ is the output estimation error. Here

$$
\operatorname{sign}\left(e_{y}\right)= \begin{cases}+1 & \text { if } e_{y}>0 \\ -1 & \text { otherwise }\end{cases}
$$

Notice that without the last term in (3) the equations have a traditional Kalman filter/Luenberger observer structure, i.e., a model of the plant driven by signals depending on the output estimation error.

When the initial conditions of the true states and observer states are deliberately set to different values, the following simulation results can be obtained. Figure 1 shows the outputs of the plant and the observer. It can be seen that that of the observer quickly tracks the output of the plant.

Figure 2 shows that a sliding motion takes place after 0.2 seconds, i.e., $e_{y}$ is forced to zero and remains at zero for all subsequent time despite the presence of uncertainty. The figure demonstrates the finite time response that is a characteristic of sliding modes.

Figure 3 shows the states of the observer and the plant. Although the difference between the output of the plant and the observer becomes zero in finite time, the state estimation error persists, although it decays to zero asymptotically despite the plant/observer mismatch (since the sine term has been ignored for the purpose of observer design).

Figure 4 shows a low pass filtered version of the nonlinear injection $\nu$. The key issue to notice in Fig. 4 is that, on average, the nonlinear term $\nu=2 \operatorname{sign}\left(e_{y}\right)$ replicates the 'unknown signal' $\xi$ without any knowledge of the signal beyond a bound on its magnitude.
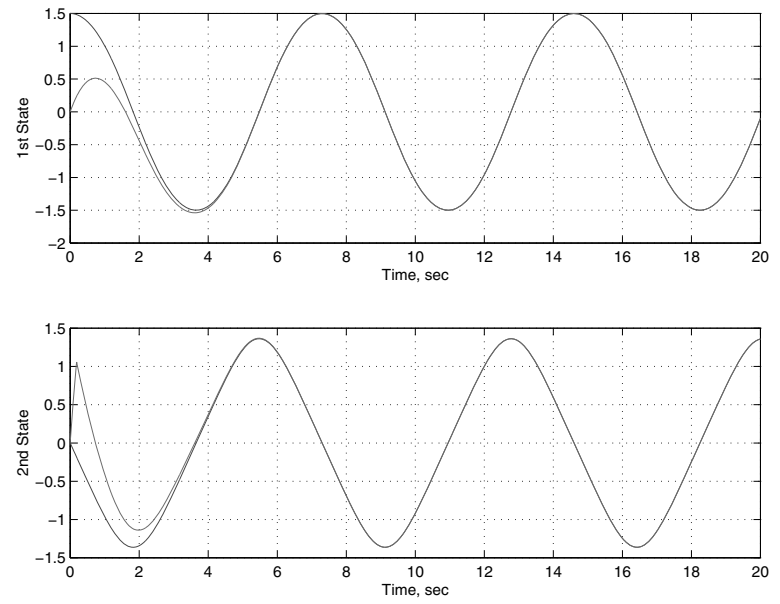

Fig. 3. Comparison of the states of the observer and the plant.

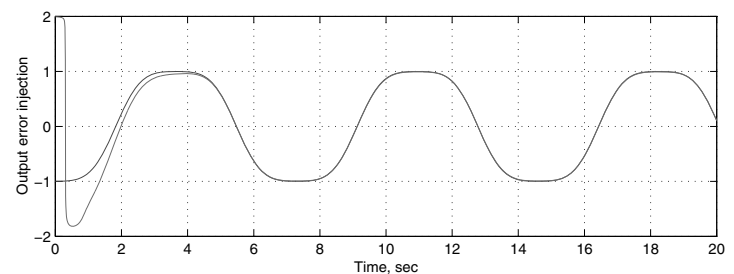

Fig. 4. Evolution of the 'equivalent output error injection' of the observer.

\section{Sliding mode observers for fault detection}

This section considers the use of sliding mode observers for fault detection. A relevant model of the problem may be posed as

$$
\begin{aligned}
& \dot{x}=A x+Q \xi(x, t)+M f_{i}(u, t), \\
& y=C x,
\end{aligned}
$$

where $A \in \mathbb{R}^{n \times n}, Q \in \mathbb{R}^{n \times h}, M \in \mathbb{R}^{n \times q}$ and $C \in$ $\mathbb{R}^{p \times n}$. The state $x(t)$ is assumed to be unknown. The bounded unknown function $f_{i}(u, t)$ represents the actuator fault to be estimated. The term $\xi(x, t)$ represents bounded uncertainty affecting the system and the fault is assumed to satisfy

$$
\left\|f_{i}(u, t)\right\| \leq k_{1}+\alpha(t, u, y),
$$

where $k_{1}$ is a positive scalar and $\alpha(\cdot)$ is a known function. The aim is to design an observer of the form

$$
\dot{z}(t)=A z(t)+B u(t)-G_{l} e_{y}(t)+G_{n} \nu,
$$


where

$$
\nu=-\rho(t, u, y) \frac{e_{y}(t)}{\left\|e_{y}(t)\right\|} \quad \text { if } e_{y}(t) \neq 0
$$

and $e_{y}(t)=\hat{y}(t)-y(t)$. The two gains $G_{l}, G_{n} \in \mathbb{R}^{n \times p}$ are to be determined and the modulation function $\rho: \mathbb{R}_{+} \times$ $\mathbb{R}^{p} \times \mathbb{R}^{m} \rightarrow \mathbb{R}_{+}$is chosen to satisfy

$$
\rho(t, y, u) \geq k_{1}+\alpha(t, u, y)+\eta,
$$

where $\eta \in \mathbb{R}_{+}$. A fixed gain $W \in \mathbb{R}^{q \times p}$ will also be sought to form a reconstruction signal

$$
\hat{f}_{i}(t)=W \nu(t) .
$$

Under the following assumptions:

A1: $C M$ has $\operatorname{rank} q$;

A2: $(A, M, C)$ is minimum phase;

the gains $G_{l}$ and $G_{n}$ can be chosen so that $\mathcal{R}(M) \subset$ $\mathcal{R}\left(G_{n}\right)$ and the transfer function $C\left(s I-A+G_{l} C\right)^{-1} G_{n}$ is strictly positive real. As a result, the signal $\hat{f}_{i}$ in (10) can be designed to have the following properties:

- if $\xi=0$, then $\hat{f}_{i} \rightarrow f_{i}$ (at worst asymptotically);

- if $\xi \neq 0$, then there exists a positive scalar $\gamma$ such that

$$
\int_{0}^{\infty}\left\|f_{i}(t)-\hat{f}_{i}(t)\right\|^{2} \mathrm{~d} t \leq \gamma^{2} \int_{0}^{\infty}\|\xi(t)\|^{2} \mathrm{~d} t
$$

where $\gamma$ represents the $\mathcal{L}_{2}$ gain between the uncertainty/disturbance $\xi$ and the fault estimation error (Tan and Edwards, 2003).

Remark 1. This is a fault estimation approach, i.e., not residual based. Moreover, provided the gain $\gamma$ is small, isolation is inherent in the scheme.

As a result of $\mathrm{A} 1$ and $\mathrm{A} 2$, there exists a change of coordinates such that

$$
\begin{aligned}
A & =\left[\begin{array}{ll}
A_{11} & A_{12} \\
A_{21} & A_{22}
\end{array}\right], & M & =\left[\begin{array}{c}
0 \\
M_{o}
\end{array}\right], \\
Q & =\left[\begin{array}{l}
Q_{1} \\
Q_{2}
\end{array}\right], & C & =\left[\begin{array}{ll}
0 & T
\end{array}\right],
\end{aligned}
$$

where $A_{11} \in \mathbb{R}^{(n-p) \times(n-p)}, M_{o} \in \mathbb{R}^{q \times q}$ is nonsingular and $T \in \mathbb{R}^{p \times p}$ is orthogonal (Edwards and Spurgeon, 1998).

Define $A_{211}$ as the top $p-q$ rows of $A_{21}$. It can be shown that $\left(A_{11}, A_{211}\right)$ is detectable. Furthermore, the unobservable modes are the invariant zeros of $(A, M, C)$ (Edwards and Spurgeon, 1998). It can be shown that a suitable choice of the gain $G_{n}$ is

$$
G_{n}=\left[\begin{array}{c}
L T^{\mathrm{T}} \\
T^{\mathrm{T}}
\end{array}\right]
$$

where

$$
L=\left[\begin{array}{ll}
L_{o} & 0
\end{array}\right]
$$

with $L_{o} \in \mathbb{R}^{(n-p) \times(p-q)}$, and

$$
\hat{f}_{i}=f_{i}+\hat{G}(s) \xi
$$

where

$$
\begin{aligned}
\hat{G}(s):= & W A_{21}\left(s I-\left(A_{11}+L A_{211}\right)^{-1}\left(Q_{1}+L Q_{21}\right)\right. \\
& +W Q_{2},
\end{aligned}
$$

where $Q_{21}$ represents the top $p-q$ rows of $Q_{2}$. The objective is to minimize the effect of $\xi$ on $\hat{f}_{i}$ in an $\mathcal{L}_{2}$ sense as in (11), with respect to the choice of $L$ and $W$. The synthesis of the observer design parameters can be posed as a convex optimization problem and solved using LMI techniques in a systematic way (Tan and Edwards, 2003). If 'precise' fault reconstruction is not possible, the LMI optimization seeks to minimize the effect of the uncertainty on the reconstruction.

Remark 2. In this paper, a clear distinction is made between faults and disturbances. The faults are to be reconstructed as accurately as possible, but there is no requirement per se to estimate the disturbances. Other works have not made this distinction. For example, Saif and Guan (1993) aggregate the faults and disturbances to form an augmented 'fault' vector and suggest using a linear unknown input observer to reconstruct the new 'fault' vector. A necessary condition in the works of Edwards et al. (2000), Edwards and Spurgeon (2000), Tan and Edwards (2003) as well as Saif and Guan (1993) is that the first Markov parameter of the system connecting the fault to the output must be full rank (i.e., Assumption A1). This limits the class of systems to which the results of Edwards et al. (2000), Edwards and Spurgeon (2000), Tan and Edwards (2003) as well as Saif and Guan (1993) are applicable.

Recently, fault reconstruction schemes for systems for which $C M$ is not full rank have been developed. Higher order sliding mode schemes have been suggested by Bejarano et al. (2007), Chen and Saif (2007), Fridman et al. (2007), Dávila et al. (2010) as well as Moreno and Osorio (2008). The work of Fridman et al. (2007) uses the notion of 'strong observability' together with the socalled higher order sliding mode observers. Strong observability concepts have also been exploited by Bejarano et al. (2007) using a hierarchy of observers. Chen and Saif (2007) advocate a bank of high-order sliding-mode differentiators to obtain derivatives of the outputs and then estimate the faults from these signals. Floquet et al. (2007) suggest the use of exact differentiators to generate derivatives of the measurements to 'create' additional outputs to circumvent relative degree assumptions. 
The problem of input reconstruction has also been considered from a geometric perspective by Edelmayer et al. (2004). The works of Chen and Saif (2007), Floquet et al. (2007), Bejarano et al. (2007), or Fridman et al. (2007) do not consider uncertainty, unless the faults and uncertainty are augmented and treated as 'unknown inputs.' In this case the number of disturbances plus faults must not exceed that of outputs. This limits the class of systems for which the results are applicable. $\mathrm{Ng}$ et al. (2007) extended the work of Tan and Edwards (2003) exploiting two sliding mode observers in cascade. Known signals from the first observer were considered as outputs of a 'fictitious' system which has a full rank (first) Markov parameter. Then a second sliding mode observer is designed based on the fictitious system to reconstruct the fault. This enables robust fault reconstruction for systems where the number of disturbances and faults exceeds that of outputs. The next section builds on the results of $\mathrm{Ng}$ et al. (2007) using multiple observers in cascade.

\section{Cascade based robust fault reconstruction scheme}

The use of sliding mode observers in a cascade framework for unknown input estimation is not new (see, e.g., Sharam and Aldeen, 2007; Wang et al., 2003; Haskara et al., 1998; Krasnova et al., 2001). However, the work of Haskara et al. (1998) assumes full state measurement, whilst Wang et al. (2003) do not consider any external disturbances. Although Sharam and Aldeen (2007) consider both faults and uncertainties, they are aggregated and both treated as unknown inputs-this introduces unnecessary conservatism.

In this section the faults and disturbances are treated differently. Using similar techniques as $\mathrm{Ng}$ et al. (2007) did, measurable signals from an observer are used as outputs of a fictitious system. The next observer is designed for the fictitious system, and the known signals from this observer are used as outputs of another fictitious system. The process is repeated until a fictitious system is obtained, whose (first) Markov parameter is full rank. The technique proposed by Tan and Edwards (2003) is then used to robustly reconstruct the fault. This results in a robust fault scheme reconstruction applicable to a wider class of systems than in the work of $\mathrm{Ng}$ et al. (2007).

The final fictitious system is found to be in the same framework as in the case of Tan and Edwards (2003), which minimizes the $\mathcal{L}_{2}$ gain from the disturbances to the fault reconstruction. This means the algorithm is applicable for systems where the number of outputs is less than the sum of the faults and disturbance channels. In addition, it is found that the design of previous observers does not affect the sliding motion of the final observer, which implies that the $\mathcal{L}_{2}$ gain from the disturbances to the fault reconstruction is not affected (Tan and Edwards, 2010).
The recursive scheme will now be described. First, re-write the system in (4)-(5) as

$$
\begin{aligned}
& \dot{x}^{1}=A^{1} x^{1}+M^{1} f^{1}+Q^{1} \xi^{1}, \\
& y^{1}=C^{1} x^{1},
\end{aligned}
$$

where $x^{1} \in \mathbb{R}^{n^{1}}$ are the states, $y^{1} \in \mathbb{R}^{p}$ are the outputs and $f^{1} \in \mathbb{R}^{q}$ are unknown faults. The signals $\xi^{1} \in \mathbb{R}^{h}$ are uncertainties that represent the mismatch between the linear model (17) and the real plant. Assume without loss of generality that $\operatorname{rank}\left(M^{1}\right)=q, \operatorname{rank}\left(C^{1}\right)=p$ and $\operatorname{rank}\left(C^{1} M^{1}\right)=\bar{r}^{1}<q$, which implies that $\bar{r}^{1} \leq$ $\min \{p, q\}$. The objective is to reconstruct $f^{1}$ whilst minimizing the effects of $\xi^{1}$ on the fault reconstruction. If $h+q>p$ and $\bar{r}^{1}<q$, then the approaches suggested by Edwards et al. (2000), Edwards and Spurgeon (2000), Saif and Guan (1993), Tan and Edwards (2003), Sharam and Aldeen (2007), Bejarano et al. (2007), Chen and Saif (2007), Fridman et al. (2007) as well as Floquet et al. (2007) are not applicable. In this situation, the following proposes the cascade observer scheme.

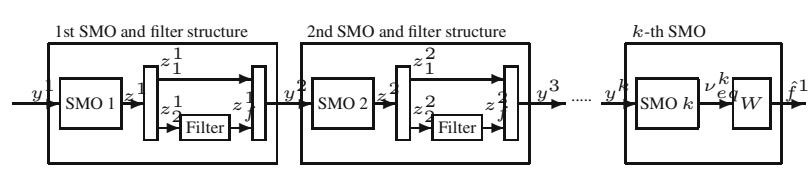

Fig. 5. Observer scheme.

For the algorithm which will be described in the sequel, partition the matrices from (17) as

$$
\begin{array}{rlr}
A^{1} & =\left[\begin{array}{ll}
A_{1}^{1} & A_{2}^{1} \\
A_{3}^{1} & A_{4}^{1}
\end{array}\right], & M^{1}=\left[\begin{array}{c}
M_{1}^{1} \\
M_{2}^{1}
\end{array}\right], \\
Q^{1} & =\left[\begin{array}{l}
Q_{1}^{1} \\
Q_{2}^{1}
\end{array}\right] \begin{array}{l}
\left\lceil n^{1}-p\right. \\
\uparrow p,
\end{array}
\end{array}
$$

where $A_{1}^{1}$ is square. Since $C^{1}=\left[\begin{array}{cc}0 & I_{p}\end{array}\right]$ and $\operatorname{rank}\left(C^{1} M^{1}\right)=\bar{r}^{1}$, we have $\operatorname{rank}\left(M_{2}^{1}\right)=\bar{r}^{1}$. In the above, $Q^{1}$ has no particular structure. The idea is to create a systematic way of

- computing the number of observers required,

- calculating the gains of the sliding mode observers.

Consider a recursive sequence of 'systems' of the form

$$
\dot{x}^{i}=A^{i} x^{i}+M^{i} f^{i}+Q^{i} \xi^{i}, \quad y^{i}=C^{i} x^{i},
$$

where $x^{i} \in \mathbb{R}^{n^{i}}$ are the states, $y^{i} \in \mathbb{R}^{p^{i}}$ the outputs and $f^{i} \in \mathbb{R}^{q}$ are unknown faults to be estimated. The signals $\xi^{i} \in \mathbb{R}^{h}$ are uncertainties. The following proposition underpins the strategy. 
Proposition 1. (Tan and Edwards, 2010) Assume that $\operatorname{rank}\left(C^{i} M^{i}\right)=\bar{r}^{i}<q^{i}$ where $q^{i}=\operatorname{rank}\left(M^{i}\right)$. Then there exists a change of coordinates $x^{i} \mapsto T_{1}^{i} x^{i}$ and $a$ nonsingular scaling $f^{i} \mapsto f^{i+1}:=T_{2}^{i} f^{i}$ such that

- the fault matrix has the structure

$$
M^{i}=\left[\begin{array}{l}
M_{1}^{i} \\
M_{2}^{i}
\end{array}\right]=\left[\begin{array}{cc}
M_{11}^{i} & 0 \\
0 & 0 \\
0 & \bar{M}_{22}^{i}
\end{array}\right] \begin{aligned}
& \uparrow n^{i}-p^{i} \\
& \uparrow \bar{r}^{i}-\bar{r}^{i}
\end{aligned},
$$

where $\bar{M}_{22}^{i} \in \mathbb{R}^{\bar{r}^{i} \times \bar{r}^{i}}$ is invertible with $M_{11}^{i}$ being full column rank;

- the output matrix has the structure

$$
C^{i}=\left[\begin{array}{ll}
0 & C_{2}^{i}
\end{array}\right],
$$

where $C_{2}^{i} \in \mathbb{R}^{p^{i} \times p^{i}}$ and is full rank;

- the matrices $A^{i}, Q^{i}$ have no particular structure but are partitioned as

$$
A^{i}=\left[\begin{array}{ll}
A_{1}^{i} & A_{2}^{i} \\
A_{3}^{i} & A_{4}^{i}
\end{array}\right], \quad Q^{i}=\left[\begin{array}{l}
Q_{1}^{i} \\
Q_{2}^{i}
\end{array}\right] \begin{aligned}
& \left\lceil n^{i}-p^{i}\right. \\
& \left\lceil p^{i}\right.
\end{aligned} .
$$

At Step $i$ suppose that $\operatorname{rank}\left(C^{i} M^{i}\right)=\bar{r}^{i}<q^{i}$, where $q^{i}=\operatorname{rank}\left(M^{i}\right)$. This is certainly true when $i=1$, otherwise the method proposed by Tan and Edwards (2003) can be used directly.

A key assumption is that $\xi$ is smooth and an upper bound on its bandwidth is known. As a result, write

$$
\xi^{1}=\Omega(s) \xi^{k},
$$

where $\Omega(s)$ is a known filter with low-pass characteristics of appropriate bandwidth and $\xi^{k}$ is a bounded unknown signal. The transfer function matrix $\Omega(s)$ can be viewed as a 'weighting function' often used in frequency domain approaches to control (Zhou et al., 1996). Furthermore, assume that each $\xi^{i}$ satisfies

$$
\dot{\xi}^{i}=A_{\Omega}^{i} \xi^{i}+B_{\Omega}^{i} \xi^{i+1},
$$

where $A_{\Omega}^{i}$ is a stable matrix and where, by definition, $\xi^{1}:=\xi$. Suitable choices for $A_{\Omega}^{i}$ and $B_{\Omega}^{i}$ need to be made to capture the characteristics of $\xi^{k}$. The idea is then to augment (19) and (24) to obtain

$$
\dot{\bar{x}}^{i}=\bar{A}^{i} \bar{x}^{i}+\bar{M}^{i} f^{i}+\bar{Q}^{i} \xi^{i+1}, \quad y^{i}=\bar{C}^{i} \bar{x}^{i} .
$$

For each intermediate system (25), an observer of the form

$$
\dot{\bar{z}}^{i}=\bar{A}^{i} \bar{z}^{i}-\bar{G}_{l}^{i} \bar{e}_{y}^{i}+\bar{G}_{n}^{i} \bar{\nu}^{i}
$$

is used, where $\bar{z}^{i} \in \mathbb{R}^{\bar{n}}$ is the estimate of $\bar{x}^{i}$ and $\bar{e}_{y}^{i}=$ $\bar{C}^{i} \bar{z}^{i}-y^{i}$. The matrices $\bar{G}_{l}^{i}, \bar{G}_{n}^{i} \in \mathbb{R}^{\bar{n}^{i} \times p^{i}}$ are observer gains (to be designed). Structurally this is the observer from (7). In the canonical form coordinates associated with Proposition 1

$$
\bar{G}_{n}^{i}=\left[\begin{array}{c}
-\bar{L}^{i} \\
I_{p}
\end{array}\right]\left(\bar{P}_{o} \bar{C}_{2}\right)^{-1}, \quad \bar{L}^{i}=\left[\begin{array}{cc}
\bar{L}_{o}^{i} & 0
\end{array}\right],
$$

where $\bar{P}_{o} \in \mathbb{R}^{p^{i} \times p^{i}}$ is semi-positive definite and $\bar{L}_{o}^{i} \in$ $\mathbb{R}^{\left(\bar{n}^{i}-p^{i}\right) \times m^{i+1}}$. The term $\bar{\nu}^{i}$ is a nonlinear discontinuous term defined by

$$
\bar{\nu}^{i}=-\bar{\rho} \frac{\bar{e}_{y}^{i}}{\left\|\bar{e}_{y}^{i}\right\|}, \bar{\rho} \in \mathbb{R}_{+} \quad \text { for } \bar{e}_{y}^{i} \neq 0 .
$$

If the modulation function $\rho$ is chosen to ensure a sliding motion, then, during sliding, in appropriate coordinates

$$
\begin{aligned}
\dot{\bar{e}}_{1}^{i} & =\left(\bar{A}_{1}^{i}+\bar{L}_{o}^{i} \bar{A}_{31}^{i}\right) \bar{e}_{1}^{i}-\bar{M}_{1}^{i} f^{i+1}-\bar{Q}_{1}^{i} \xi^{i+1}, \\
0 & =\bar{C}_{2}^{i} \bar{A}_{3}^{i} \bar{e}_{1}^{i}-\bar{C}_{2}^{i} \bar{M}_{2}^{i} f^{i+1}+\left(\bar{P}_{o}^{i}\right)^{-1} \bar{\nu}_{e q}^{i},
\end{aligned}
$$

where $\bar{\nu}_{e q}^{i}$ is the equivalent output injection. Making a change of variables $w^{i}:=-\bar{e}_{1}^{i}$ and re-arranging (29)- (30) gives the representation

$$
\begin{aligned}
\dot{w}^{i}= & \left(\bar{A}_{1}^{i}+\bar{L}_{o}^{i} \bar{A}_{31}^{i}\right) w^{i}+\bar{M}_{1}^{i} f^{i+1} \\
& +\bar{Q}_{1}^{i} \xi^{i+1} \\
\left(\bar{P}_{o}^{i} \bar{C}_{2}^{i}\right)^{-1} \bar{\nu}_{e q}^{i}= & \bar{A}_{3}^{i} w^{i}+\bar{M}_{2}^{i} f^{i+1},
\end{aligned}
$$

Define

$$
z^{i}:=\left(\bar{P}_{o}^{i} \bar{C}_{2}^{i}\right)^{-1} \bar{\nu}_{e q}^{i}=\left[\begin{array}{l}
z_{1}^{i} \\
z_{2}^{i}
\end{array}\right] \begin{aligned}
& \uparrow m^{i+1} \\
& { }^{i+1}-m^{i+1}
\end{aligned} .
$$

Then in a suitable coordinate system,

$$
\begin{aligned}
z_{1}^{i} & =\left[\begin{array}{ll}
0 & I_{m^{i+1}}
\end{array}\right] w^{i}, \\
z_{2}^{i} & =\bar{A}_{32}^{i} w^{i}+\left[\begin{array}{cc}
0 & 0 \\
0 & \bar{M}_{22}^{i}
\end{array}\right] f^{i+1} .
\end{aligned}
$$

Define a signal $z_{f}^{i}$ (a filtered version of $z_{2}^{i}$ ) such that

$$
\dot{z}_{f}^{i}:=-\alpha^{i} z_{f}^{i}+\alpha^{i} z_{2}^{i},
$$

where $\alpha^{i} \in \mathbb{R}_{+}$. From Eqns. (34) and (35),

$$
\dot{z}_{f}^{i}=-\alpha^{i} z_{f}^{i}+\alpha^{i} \bar{A}_{32}^{i} w^{i}+\left[\begin{array}{cc}
0 & 0 \\
0 & \alpha^{i} \bar{M}_{22}^{i}
\end{array}\right] f^{i+1} .
$$

Combining (31), 33) and 36 yields the state-space system representation

$$
\begin{aligned}
& \dot{x}^{i+1}=A^{i+1} x^{i+1}+M^{i+1} f^{i+1}+Q^{i+1} \xi^{i+1}, \\
& y^{i+1}=C^{i+1} x^{i+1},
\end{aligned}
$$

where $x^{i+1}:=\operatorname{col}\left(w^{i}, z_{f}^{i}\right), y^{i+1}:=\operatorname{col}\left(z_{1}^{i}, z_{f}^{i}\right)$ and

$$
\begin{aligned}
A^{i+1} & :=\left[\begin{array}{cc}
\bar{A}_{1}^{1}+\bar{L}_{o}^{1} \bar{A}_{31}^{1} & 0 \\
\alpha^{1} \bar{A}_{32}^{1} & -\alpha^{1} I
\end{array}\right], \\
M^{i+1} & =\left[\begin{array}{c}
\bar{M}_{1}^{1} \\
{\left[\begin{array}{cc}
0 & 0 \\
0 & \alpha^{1} \bar{M}_{22}^{1}
\end{array}\right]}
\end{array}\right],
\end{aligned}
$$


where $Q^{i+1}=\operatorname{col}\left(\bar{Q}_{1}^{1}, 0\right)$ and $C^{i+1}=\left[\begin{array}{ll}0 & I_{p}\end{array}\right]$.

Notice that $(38)$ is in the form of (19). Now only two scenarios can occur:

- $\operatorname{rank}\left(C^{i+1} M^{i+1}\right)<\operatorname{rank}\left(M^{i+1}\right)$ and the process continues with $i \leftarrow i+1$.

- $\operatorname{rank}\left(C^{i+1} M^{i+1}\right)=\operatorname{rank}\left(M^{i+1}\right)$ and a sliding mode observer of the type as in the work of Tan and Edwards (2003) based on $A^{i+1}, M^{i+1}, C^{i+1}, Q^{i+1}$ can be used to reconstruct $f^{i+1}$ and also minimize the $\mathcal{L}_{2}$ gain from $\xi^{i+1}$ to the fault reconstruction. (2010):

Key results can be stated following Tan and Edwards

- If $(A, M, C)$ is minimum phase, then all the fictitious systems $\left(A^{i}, M^{i}, C^{i}\right)$ are minimum phase. (This guarantees the existence of stable sliding motions.)

- The gain matrix $\bar{L}^{i-1}$ affects only the last $p$ columns of $A^{i}$, and it can be shown that $\bar{L}^{i-1}$ will not affect the reduced order sliding motion of observer $i$ and all subsequent observers.

Therefore, the quality of the fault reconstruction depends on the sliding motion of the last observer $i=k$.

Remark 3. The choice of the filter in 24) is important to capture the characteristics of the uncertainty $\xi^{k}$. The choice of the filters $\left(A_{\Omega}^{i}, B_{\Omega}^{i}\right)$ is not unique. The crucial decision is the choice of the filter bandwidth and not the particular choice of the filter itself. In the example which follows, first order filters have been chosen, although a higher order filter could have been used. The hypothesis here is that the uncertainties $\xi^{k}$ are assumed to be smooth and an upper bound on their bandwidth known. The assumption that there is a bound on the frequency content of the disturbances is common in the applications literature. This sort of information has been used in the development of models of practical engineering systems such as, e.g., satellites and ships and for process control, (typically, the disturbances are then assumed to be of low frequency in character). Insight into the underlying physics is usually employed to decide on the meaningful frequency range of the disturbance (Tan and Edwards, 2010).

Remark 4. A common approach in terms of practical implementation of classical sliding mode schemes is to replace the unit vector terms with a sigmoidal approximation (e.g., Edwards and Spurgeon, 1998). In the cascade scheme this will lead to a loss of accuracy. Instead, the unit vector can be replaced by a super-twist scheme (Levant, 2003) term to preserve accuracy. The super-twist scheme can be included within the Lyapunov analysis as discussed by Tan and Edwards (2010).
4.1. Design example. The method described above will now be demonstrated using a model of a civil aircraft (Edwards et al., 2010) whose system matrices are given as follows:

$$
\begin{aligned}
& A^{1}=\left[\begin{array}{rrr}
-0.5137 & -0.5831 & -0.6228 \\
1.0064 & -0.6284 & -0.0352 \\
0 & 0 & -37.0000 \\
0 & 1.7171 & 0 \\
1.0000 & 0 & 0
\end{array}\right. \\
& 0.0004 \quad 0 \\
& -0.0021 \\
& 0 \\
& 0 \\
& \left.\begin{array}{rr}
-0.0166 & -9.8046 \\
0 & 0
\end{array}\right] \\
& M^{1}=\left[\begin{array}{lllll}
0 & 0 & 37 & 0 & 0
\end{array}\right]^{T},
\end{aligned}
$$

where the states are the pitch rate, angle of attack, elevator position, total airspeed and pitch angle. The input is the elevator command. It is assumed that the first and second rows of the matrix $A^{1}$ contain uncertainties associated with the aerodynamic derivatives. The problem is to reconstruct actuator faults using only measurements of the speed and pitch angle. If the signals $f^{1}$ and $\xi^{1}$ are augmented to form a new 'fault' vector, this results in a new 'fault' vector having three components.

The filter matrices that describe the characteristics of $\xi^{1}$ are chosen here as $A_{\Omega}^{1}=-10 I_{2}$ and $B_{\Omega}^{1}=10 I_{2}$. Note that this choice is not unique: first order linear filter realizations have been chosen, although higher order filters could have been used as well. The crucial decision is the choice of the filter bandwidth and not the particular choice of the filter itself. With this choice of filter, it can be shown that $C^{2} M^{2}=0$, and hence $\bar{r}^{2}=0$, which results in $r^{2}=$ 0 . The matrices of the filter associated with $\xi^{2}$ have been chosen as $A_{\Omega}^{2}=-10 I_{2}, B_{\Omega}^{2}=10 I_{2}$. It can be shown that this gives $m^{3}=1$ and $\operatorname{rank}\left(C^{3} M^{3}\right)=\operatorname{rank}\left(M^{3}\right)$, and the robust sliding mode observer can be designed based on $A^{3}, M^{3}, C^{3}, Q^{3}$ as described in Section 3

Figure 6 shows the nominal case when there is no uncertainty. Figure 7 compares the disturbances $\xi^{1}$ that impact on the system and shows $\xi^{3}$, which is the fictitious disturbance signal associated with $\xi^{1}=\Omega(s) \xi^{3}$. It can be seen that $\xi^{3}$ is visually identical to $\xi^{1}$, which implies the weighting function for the disturbance is valid. Figure 8 shows the fault reconstruction in the presence of the uncertainty. Although there is a slight degradation due to $\Delta A^{1}$, the reconstruction is not severely affected by $\xi^{1}$ (which is significant-being more than $10 \%$ of the magnitude of the fault). 


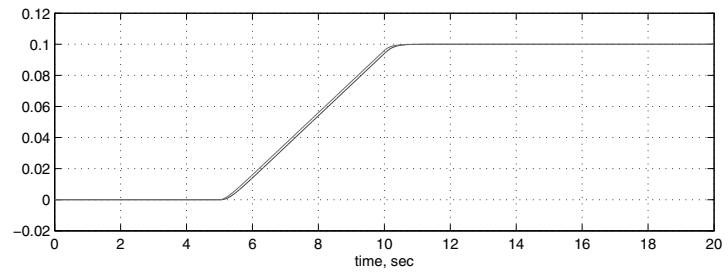

Fig. 6. Fault applied to the actuator and its reconstruction when $\Delta A^{1}=0$, i.e., when there is no uncertainty.

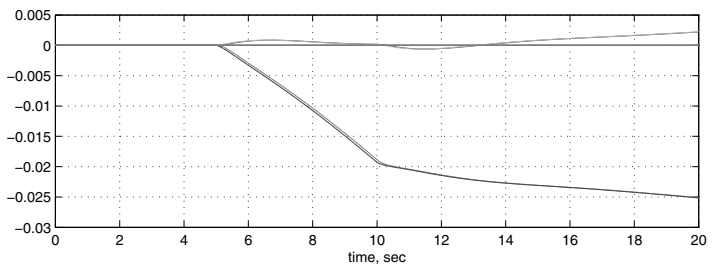

Fig. 7. Components of $\xi^{1}$ and the fictitious signal $\xi^{3}$.

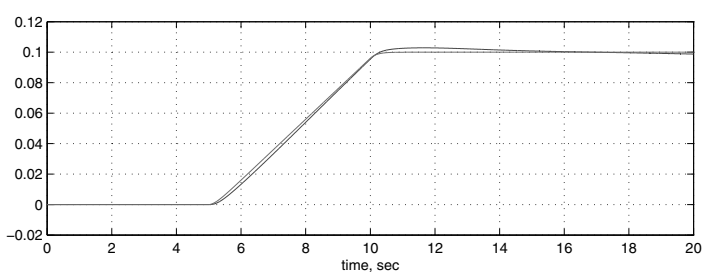

Fig. 8. Fault reconstruction in the presence of uncertainty.

\section{Reconstruction of incipient sensor faults}

Consider initially 11 a nominal dynamical system affected by sensor faults modelled as

$$
\begin{aligned}
& \dot{x}(t)=A x(t)+B u(t), \\
& y(t)=C x(t)+F f_{o}(t),
\end{aligned}
$$

where $A \in \mathbb{R}^{n \times n}, B \in \mathbb{R}^{n \times m}, C \in \mathbb{R}^{p \times n}$ and $F \in$ $\mathbb{R}^{p \times q}$, with $n \geq p>q$. The methods for sensor fault estimation proposed by Tan and Edwars $(2002 ; 2003)$ require one (testable) assumption, to guarantee the existence of the observer design. Tan and Edwards (2002) suggest introducing a new state $x_{f} \in \mathbb{R}^{p}$ satisfying

$$
\dot{x}_{f}(t)=-A_{f} x_{f}(t)+A_{f} y(t),
$$

where $-A_{f} \in \mathbb{R}^{p \times p}$ is a stable matrix. Equations (39) and (41) can be combined to give a system of order $n+p$ with states $x_{a}=\operatorname{col}\left(x_{p}, x_{f}\right)$ in the form

$$
\begin{aligned}
& \dot{x}_{a}(t)=A_{a} x_{a}(t)+B_{a} u(t)+M_{a} f_{o}(t), \\
& x_{f}(t)=C_{a} x_{a}(t),
\end{aligned}
$$

\footnotetext{
${ }^{1}$ An extension to uncertain systems is discussed by Alwi et al. (2009a).
}

It can be shown that the invariant zeros of $\left(A_{a}, M_{a}, C_{a}\right)$ are a subset of the open loop poles of the plant (cf. Tan and Edwards, 2002; 2003). A sufficient condition for using observers of the structure as in Section 2 is therefore that the system is open-loop stable in order to robustly estimate the sensor faults. Open-loop stability is not a necessary condition, but for open-loop unstable systems with certain classes of faults, examples can be constructed such that the methods given by Tan and Edwards $(2003 ; 2002)$ are not applicable. Note that classical linear Unknown Input Observers (UIOs) cannot be employed in this situation (Edwards and Tan, 2006; Chen et al., 1996; Chen and Zhang, 1991; Darouach, 1994; Saif and Guan, 1993). This section discusses a new observer design for sensor fault reconstruction which addresses this restriction.

Without loss of generality, it can be assumed that the outputs of the system have been reordered (and scaled if necessary) so that

$$
F=\left[\begin{array}{c}
0 \\
I_{q}
\end{array}\right], \quad C=\left[\begin{array}{c}
C_{1} \\
C_{2}
\end{array}\right] .
$$

The function $f_{o}: \mathbb{R}_{+} \rightarrow \mathbb{R}^{q}$ is assumed to be unknown but smooth and bounded. The objective is to design a sliding mode observer to reconstruct the faults $f_{o}(t)$ using only $y(t)$ and $u(t)$. Define

$$
\varphi(t):=\dot{f}_{o}(t) .
$$

It is assumed that the sensor faults are incipient (Patton et al., 1989) and hence $\|\varphi(t)\|$ is small, but over time the effects of the fault increment and become significant. Equations (39) and (45) can be combined to give a system of order $n+q$ with states $x_{a}:=\operatorname{col}\left(x, f_{o}\right)$ in the form

$$
\begin{aligned}
{\left[\begin{array}{c}
\dot{x} \\
\dot{f}_{o}
\end{array}\right] } & =\underbrace{\left[\begin{array}{cc}
A & 0 \\
0 & 0
\end{array}\right]}_{A_{a}}\left[\begin{array}{c}
x \\
f_{o}
\end{array}\right]+\underbrace{\left[\begin{array}{c}
B \\
0
\end{array}\right]}_{B_{a}} u+\underbrace{\left[\begin{array}{c}
0 \\
I_{q}
\end{array}\right]}_{F_{a}} \varphi, \\
y & =\underbrace{\left[\begin{array}{ll}
C & F
\end{array}\right]}_{C_{a}}\left[\begin{array}{c}
x \\
f_{o}
\end{array}\right] .
\end{aligned}
$$

Equations (46) and (47) represent an unknown input problem for $\left(A_{a}, F_{a}, C_{a}\right)$ driven by the unknown signal $\varphi(t)$.

Proposition 2. (Alwi et al., 2009b) The pair $\left(A_{a}, C_{a}\right)$ is observable if $\left(A, C_{1}\right)$ does not have an unobservable mode at zero or if the open loop system in (39) is stable.

After an appropriate change of coordinates (Alwi et al., 2009a), the triple in the new coordinates is given by

$\tilde{A}_{a}=\left[\begin{array}{cc}A & 0 \\ C_{2} A & 0\end{array}\right], \quad \tilde{C}_{a}=\left[\begin{array}{ll}0 & I_{p}\end{array}\right], \quad \tilde{F}_{a}=\left[\begin{array}{c}0 \\ I_{q}\end{array}\right]$, 
where $C_{2} \in \mathbb{R}^{q \times n}$. In the $x_{a}$ coordinates,

$$
f_{o}(t)=C_{f} x_{a}(t)
$$

where $C_{f}:=\left[\begin{array}{ll}0_{q \times n} & I_{q}\end{array}\right]$. Write

$$
A_{a}=\left[\begin{array}{c|c}
A_{11} & A_{12} \\
\hline A_{211} & A_{22} \\
A_{212} &
\end{array}\right],
$$

where the matrices $A_{11} \in \mathbb{R}^{(n+q-p) \times(n+q-p)}$ and $A_{211} \in \mathbb{R}^{(p-q) \times(n+q-p)}$. By construction, the unobservable modes of $\left(A_{11}, A_{211}\right)$ are the invariant zeros of $\left(A_{a}, F_{a}, C_{a}\right)$ (Edwards et al., 2000). For the system in (46) and (47), consider a sliding mode observer of the form given in (7) and (8). An appropriate gain $G_{n}$ for the nonlinear injection term $\nu$ in 28 is

$$
G_{n}=\left[\begin{array}{c}
-L \\
I_{p}
\end{array}\right], \quad L=\left[\begin{array}{ll}
L_{1} & L_{2}
\end{array}\right]
$$

where $L_{1} \in \mathbb{R}^{(n+q-p) \times(p-q)}$ and $L_{2} \in \mathbb{R}^{(n+q-p) \times q}$ represent design freedom (Edwards and Spurgeon, 1994). The reduced order sliding motion can be written as

$$
\begin{aligned}
& \dot{\tilde{e}}_{1}(t)=\left(A_{11}+L_{1} A_{211}+L_{2} A_{212}\right) \tilde{e}_{1}(t)+L_{2} \varphi, \\
& \dot{e}_{y}(t)=e_{y}(t)=0 .
\end{aligned}
$$

The matrices $L_{1}$ and $L_{2}$ have to be chosen to ensure that $A_{11}+L A_{211}+L_{2} A_{212}$ is stable. The effect of $\varphi$ on the estimation $\hat{f}_{o}$ is given by $\tilde{G}(s) \varphi$, where

$$
\tilde{G}(s):=\left[\begin{array}{c|c}
A_{11}+L_{1} A_{211}+L_{2} A_{212} & L_{2} \\
\hline C_{e} & 0
\end{array}\right],
$$

with $C_{e}=\left[\begin{array}{cc}0_{n-p \times q} & I_{q}\end{array}\right]$ Since the pair $\left(A_{a}, C_{a}\right)$ is observable, there exist matrices $L_{1}$ and $L_{2}$ so that the system matrix $A_{11}+L_{1} A_{211}+L_{2} A_{212}$ is stable.

Proposition 3. If $\left(A_{a}, F_{a}, C_{a}\right)$ from (39) and (40) is minimum phase, then a sliding mode observer exists such that $\hat{f}_{o}=C_{f} x_{a} \rightarrow f_{o}$ as $t \rightarrow \infty\left(\right.$ choosing $\left.L_{2}=0\right)$.

Proposition 4. If the system matrix A from (39) is stable, then a sliding mode observer exists such that $\hat{f}_{o}=$ $C_{f} z_{a} \rightarrow f_{o}$ as $t \rightarrow \infty$.

Remark 5. If $A$ from 39 is unstable, then for certain fault conditions $\left(A, C_{1}\right)$ may be unobservable and perfect reconstruction is not possible. Furthermore, if $\left(A, C_{1}\right)$ is undetectable making $\left(A_{a}, F_{a}, C_{a}\right)$ nonminimum phase, then, as argued by Edwards and Tan (2006), unknown input observers cannot be employed to reject $\varphi$, (see Saif and Guan, 1993; Darouach, 1994; Chen and Zhang, 1991; Chen et al., 1996). As described by Alwi et al. (2009a), the gains $L_{1}$ and $L_{2}$ must be chosen to ensure that $\|\tilde{G}(s)\|_{\infty}$ is minimised.
5.1. Simulation results. The ADMIRE model represents a small rigid fighter aircraft with a delta-canard configuration (Forssell and Nilsson, 2005). The linear model used for design has been obtained at a low speed flight condition similar to the one given by Härkegård and Glad (2005). The controlled outputs are angle of attack, sideslip the angle and roll rate. The linear model is open-loop unstable, which is typical for fighter aircraft to allow high manoeuvrability. It is assumed that the sensor for the pitch rate $(q)$ is prone to faults. It can be shown that the associated augmented system $\left(A_{a}, F_{a}, C_{a}\right)$ is non-minimum phase (Alwi et al., 2009a).

The simulation displayed in Figs. 9 and 10 has been obtained from the full nonlinear ADMIRE model with the aircraft undergoing a banking manoeuvre and change in altitude. Figure 10 shows the results of the fault reconstruction using different sensor fault shapes, to show the effectiveness of the method. In both conditions, the proposed scheme provides satisfactory fault reconstructions for the $q$-th sensor. As expected, perfect fault estimation cannot be achieved.

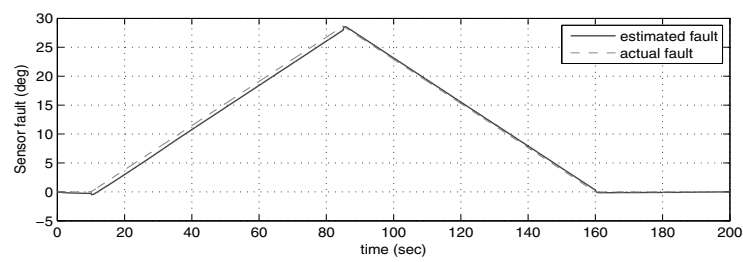

Fig. 9. Sensor fault reconstruction on the pitch rate.

\section{Fault tolerant control}

The inherent robustness properties of sliding modes to matched uncertainty make it a natural candidate for passive fault tolerant control. It is argued by Alwi and Edwards $(2008 \mathrm{a}$; 2008b) that a broad class of actuator faults can be accommodated by an appropriate scheme which monitors quantitatively the extent to which a sliding motion (in a control context) is being maintained and then triggers an adaptive mechanism if there is deterioration in performance. The controller is based around a state-

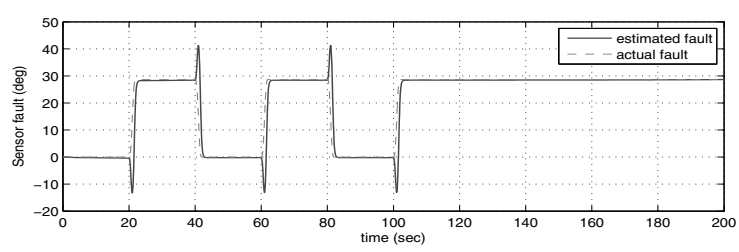

Fig. 10. Sensor fault reconstruction on the pitch rate. 
feedback sliding mode scheme and the gain associated with the nonlinear term is allowed to adaptively increase when the onset of a fault is detected. Compared with other FTC schemes which have been implemented on this model, the controller is simple and yet is shown to work across the entire 'up and away' flight envelope.

Although sliding mode controllers (e.g., Alwi and Edwards, 2008a) cope easily with faults, they are not able to directly deal with failures, i.e., the total loss of an actuator. In order to overcome this, the integration of a sliding mode scheme with a control allocation framework has been considered (Alwi and Edwards, 2008b), where the effectiveness level of the actuators is used to redistribute the control signals to the 'healthier' actuators when a fault occurs.

One of the challenges of using traditional control ideas for systems with redundancy, i.e., over-actuated systems, is how to deal with these additional degrees of freedom. Control Allocation (CA) has emerged as one of the most studied techniques when dealing with such problems (e.g., Enns, 1998; Bošković and Mehra, 2002; Buffington et al., 1999; Davidson et al., 2001). One benefit of using a CA structure for fault tolerant control is that the controller remains the same and the control effort is distributed to all available actuators without reconfiguration. This is vital in terms of simplicity of design.

Recently, Alwi and Edwards (2008b) developed a rigorous design procedure from a theoretical perspective to achieve FTC while proving stability for a class of faults and failures. Their work has been used to design lateral and longitudinal controllers for the GARTEUR FMAG16 benchmark problem (Edwards et al., 2010). The GARTEUR FM-AG16 action group has undertaken an extensive study to establish the benefits of using state of the art fault detection and FTC methods for aerospace systems. The different paradigms which have been applied are described by Edwards et al. (2010). The control allocation scheme described here uses the effectiveness levels to redistribute the control signals to functioning healthy actuators when a fault/failure occurs (Alwi and Edwards, 2008b; Alwi et al., 2008).

6.1. Design procedures. Consider an over-actuated system subject to actuator faults,

$$
\dot{x}(t)=A x(t)+B u(t)-B K u(t),
$$

where $A \in \mathbb{R}^{n \times n}$ and $B \in \mathbb{R}^{n \times m}$. The matrix $K=$ $\operatorname{diag}\left(k_{1}, \ldots, k_{m}\right)$, where the scalars $0 \leq k_{i} \leq 1$ model a decrease in effectiveness of an actuator. If $k_{i}=0$, the actuator is healthy, otherwise a fault is present, and if $k_{i}=$ 1 the actuator has failed totally. The work of Alwi and Edwards (2008b) advocates reordering the states such that

$$
B=\left[\begin{array}{l}
B_{1} \\
B_{2}
\end{array}\right]
$$

where $B_{2} \in \mathbb{R}^{l \times m}$ has rank $l$ and $\left\|B_{2}\right\|=1$ with $\left\|B_{1}\right\| \ll$ 1 . Here $l$ reflects the number of controlled outputs. Let the 'virtual control' $\nu(t):=B_{2} u(t)$ so that $u(t)=B_{2}^{\dagger} \nu(t)$, where

$$
B_{2}^{\dagger}:=W B_{2}^{\mathrm{T}}\left(B_{2} W B_{2}^{\mathrm{T}}\right)^{-1}
$$

and $W \in \mathbb{R}^{m \times m}$. Note $B_{2} B_{2}^{\dagger}=I_{l}$ for any choice of $W$. In the work of Alwi and Edwards (2008b) the choice

$$
W=I-K
$$

is suggested (assuming good estimates of $k_{i}$ are available). In a fault free situation $W=I$, which is a common choice in the CA literature. Sliding mode control methods (Utkin, 1992; Edwards and Spurgeon, 1998) will be used to synthesize $\nu(t)$. Define a switching function $\sigma(t): \mathbb{R}^{n} \rightarrow \mathbb{R}^{l}$ to be

$$
\sigma(t)=S x(t)
$$

where $S \in \mathbb{R}^{l \times n}$ and $\operatorname{det}\left(S B_{\nu}\right) \neq 0$. After an appropriate coordinate transformation $x \mapsto \hat{x}=T_{r} x$, the system can be written as

$$
\left[\begin{array}{l}
\dot{\hat{x}}_{1}(t) \\
\hat{\hat{x}}_{2}(t)
\end{array}\right]=\left[\begin{array}{ll}
\hat{A}_{11} & \hat{A}_{12} \\
\hat{A}_{21} & \hat{A}_{22}
\end{array}\right]\left[\begin{array}{l}
\hat{x}_{1}(t) \\
\hat{x}_{2}(t)
\end{array}\right]+\left[\begin{array}{c}
B_{1} B_{2}^{N} B_{2}^{+} \\
I
\end{array}\right] \hat{\nu}(t),
$$

where

$$
B_{2}^{N}:=\left(I-B_{2}^{\mathrm{T}} B_{2}\right), \quad B_{2}^{+}=W^{2} B_{2}^{\mathrm{T}}\left(B_{2} W^{2} B_{2}^{\mathrm{T}}\right)^{-1}
$$

and

$$
\hat{\nu}(t):=\left(B_{2} W^{2} B_{2}^{\mathrm{T}}\right)\left(B_{2} W B_{2}^{\mathrm{T}}\right)^{-1} \nu(t) .
$$

The following proposition is crucial:

Proposition 5. (Alwi and Edwards, 2008b) There exists a scalar $\gamma_{0}$ such that

$$
\left\|B_{2}^{+}\right\|<\gamma_{0}
$$

for all $W=\operatorname{diag}\left(w_{1}, \ldots, w_{m}\right)$ such that $0<w_{i} \leq 1$.

In the $\hat{x}(t)$ coordinates,

$$
\hat{S}:=S T_{r}^{-1}=\left[\begin{array}{ll}
N & I
\end{array}\right],
$$

where $N \in \mathbb{R}^{l \times(n-l)}$ represents design freedom. If $\left(A, B_{\nu}\right)$ is controllable, then $\left(\hat{A}_{11}, \hat{A}_{12}\right)$ is controllable and $N$ can be chosen to make $\hat{A}_{11}-\hat{A}_{12} N$ stable. The sliding motion is governed by

$$
\dot{\hat{x}}_{1}(t)=\left(\tilde{A}_{11}-B_{1} B_{2}^{N} B_{2}^{+}\left(I+N B_{1} B_{2}^{N} B_{2}^{+}\right)^{-1} \tilde{A}_{21}\right) \hat{x}_{1}(t) .
$$

In fault free conditions, $\left.B_{2}^{N} B_{2}^{+}\right|_{W=I}=0$, and the system in 63. 'collapses' to

$$
\dot{\hat{x}}_{1}(t)=\tilde{A}_{11} \hat{x}_{1}(t) .
$$


The system in (63) depends on $W$ and stability needs to be established. Define

$$
\tilde{G}(s):=\tilde{A}_{21}\left(s I-\tilde{A}_{11}\right)^{-1} B_{1} B_{2}^{N},
$$

where $\tilde{A}_{11}=\hat{A}_{11}-\hat{A}_{12} N$ and $\tilde{A}_{21}:=N \tilde{A}_{11}+\hat{A}_{21}-\hat{A}_{22}$. By construction, $\tilde{G}(s)$ is stable.

Define $\gamma_{2}=\|\tilde{G}(s)\|_{\infty}$ and

$$
\gamma_{1}:=\left\|M B_{1} B_{2}^{N}\right\| \text {. }
$$

The following proposition provides stability guarantees for the closed-loop fault system.

Proposition 6. (Alwi and Edwards, 2008b) During a fault or failure condition, for any $0<w_{i} \leq 1$, the closed-loop system will be stable if

$$
0<\frac{\gamma_{2} \gamma_{0}}{1-\gamma_{1} \gamma_{0}}<1
$$

where $\gamma_{0}=\left\|B_{2}^{+}\right\|$as defined in Proposition 5 .

The proposed control law is

$$
\hat{\nu}(t)=\hat{\nu}_{l}(t)+\hat{\nu}_{n}(t),
$$

where $\hat{\nu}_{l}(t):=-\tilde{A}_{21} \hat{e}_{1}(t)-\tilde{A}_{22} \sigma(t)$ and

$$
\hat{\nu}_{n}(t):=-(\rho(t, x)+\eta) \frac{\sigma(t)}{\|\sigma(t)\|} \quad \text { for } \sigma(t) \neq 0 .
$$

The gain from 67 is

$$
\rho(t)=r(t)\left(l_{1}\|x(t)\|+l_{2}\right) .
$$

The scalar variable $r(t)$ is an adaptive gain satisfying

$$
\dot{r}(t)=a\left(l_{1}\|x(t)\|+l_{2}\right) D_{\epsilon}(\|\sigma(t)\|)-b r(t),
$$

where $r(0)=0$, and the $a$ and $b$ are design constants. The function $D_{\epsilon}: \mathbb{R} \mapsto \mathbb{R}$ is the nonlinear function

$$
D_{\epsilon}(\|\sigma\|)= \begin{cases}0 & \text { if }\|\sigma\|<\epsilon, \\ \|\sigma\| & \text { otherwise }\end{cases}
$$

where $\epsilon$ is a positive scalar. The idea is only to trigger the adaptive scheme if a fault is present and a degradation in the sliding motion begins to appear (Alwi et al., 2010).

\section{Implementation results}

7.1. Actuator faults. The SIMONA (SImulation, MOtion and NAvigation) simulator is a motion simulator developed by the Delft University of Technology. The flight deck provides pilots with simulated instruments. The pilot interfaces with the 'aircraft' by a traditional control column or a sidestick controller, rudder pedals with engine controls and a Mode Control Panel (MCP). The windows

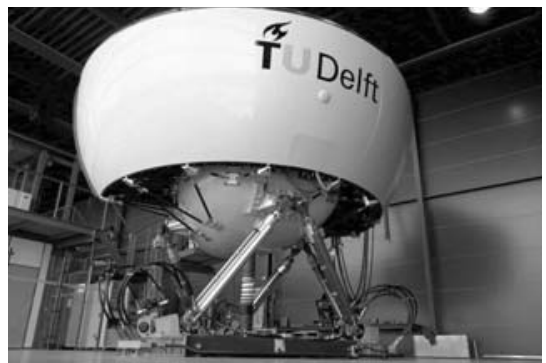

Fig. 11. SIMONA flight simulator at the TU Delft.

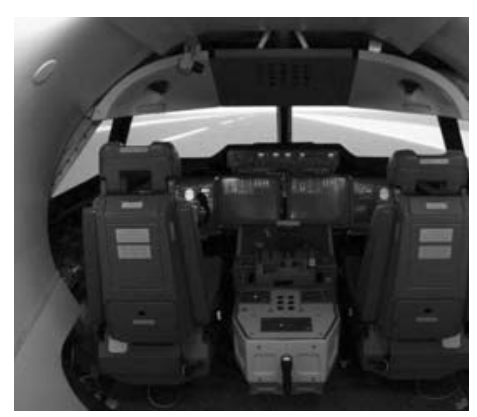

Fig. 12. SIMONA flight deck.

give a view of a virtual environment and a motion system moves the entire cabin to simulate aircraft motion. A network of PCs provides the processing power to run the simulator. A flexible software architecture (DUECCA) allows the integration of the controller in a realistic aircraft environment.

The design objective is to bring a faulty aircraft to near landing conditions. This is achieved by tracking:

- roll $(\phi)$ and sideslip $(\beta)$ using the lateral controller,

- Flight Path Angle (FPA) and airspeed ( $\left.V_{\text {tas }}\right)$ commands using the longitudinal controller.

The lateral control surfaces are the ailerons (four), spoilers (ten) and EPR (differential). The longitudinal control surfaces are the elevator, horizontal stabilizer and total EPR.

The controller was implemented using Matlab's RTW utility. The Ode4 solver with a fixed time step of $0.01 \mathrm{~s}$ was employed running on a dual Pentium III $1 \mathrm{GHz}$ processor. A connection with the MCP enables the selection of 'control modes', e.g., altitude hold, heading select, etc.

Figures 14 and 15 show the responses in the face of a horizontal stabilizer runaway, where the stabilizer moves at its maximum deflection rate to its maximum deflection 


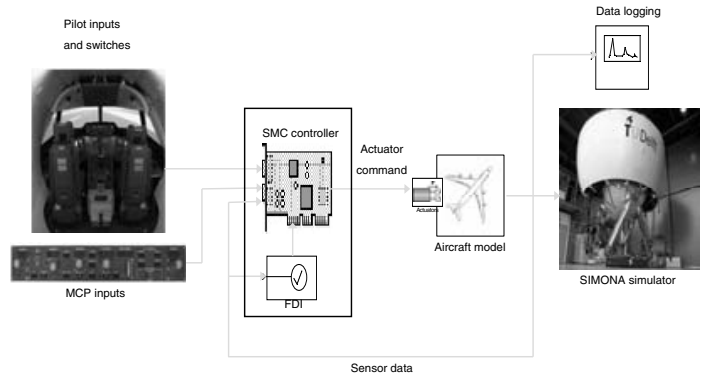

Fig. 13. Schematic of the SIMONA setup.

position of $3 \mathrm{deg}$. The aircraft performs a series of 90 degree turns after the fault has occurred followed by an attempt to reduce altitude to bring the aircraft to near landing conditions. It can be seen in Figs. 14 and 15 that good performance can be maintained despite the severe failure which has occurred in the system.
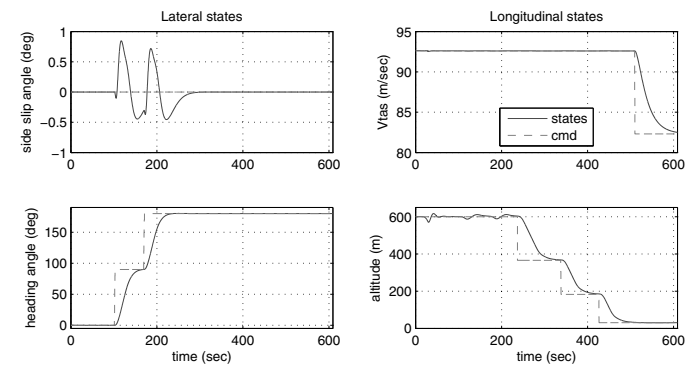

Fig. 14. Horizontal stabilizer runaway fault.
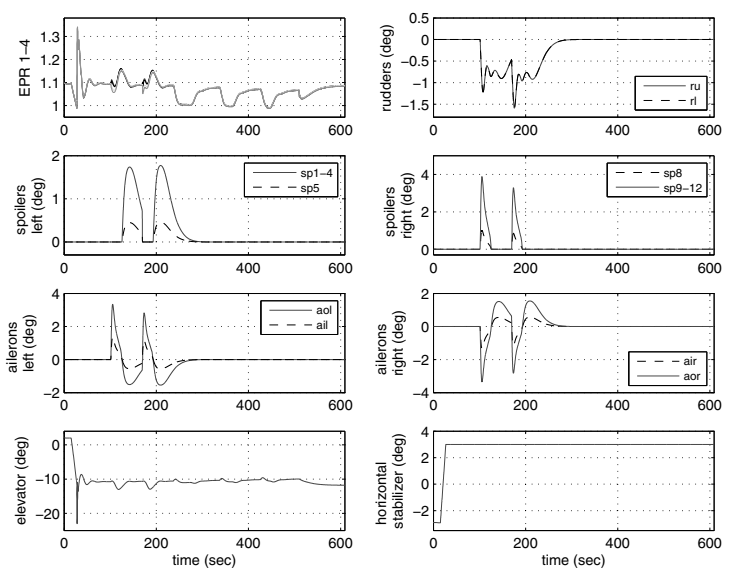

Fig. 15. Horizontal stabilizer runaway (control signal).

Figures 16 and 17 are associated with a lateral fault, specifically, a rudder runaway. The aircraft is piloted to follow the same flight path as in Figs. 14 and 15 Again it can be seen from Figs. 16 and 17 that good performance can be maintained and the aircraft can be brought to a near landing condition.
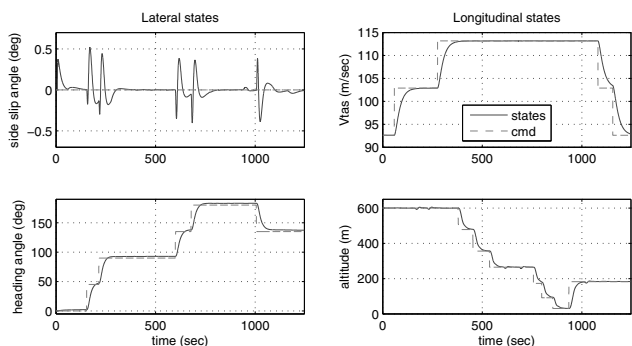

Fig. 16. Rudder runaway.
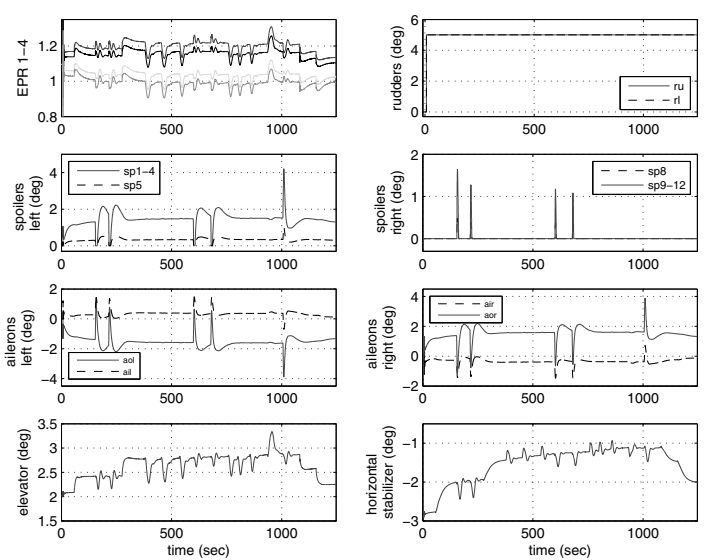

Fig. 17. Rudder runaway (control signal).

7.2. Sensor faults. Now a sensor fault scenario is considered. A general configuration representing the proposed sensor FTC scheme is given in Fig. 18. The out-

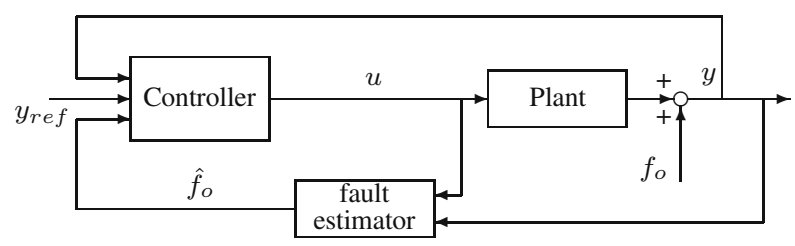

Fig. 18. Sensor fault tolerant control scheme.

put of the FDI module is the sensor fault estimate $\hat{f}_{o}$. The estimated sensor fault $\hat{f}_{o}$ will be used to correct the measured output signal, and $y-\hat{f}_{o}$ will be used in the control law calculations to generate $u$. The controller is designed for an 'up and away' flight envelope with Flight Path Angle (FPA) and true airspeed $\left(V_{\text {tas }}\right)$ as controlled outputs. A nominal fault-free sliding mode controller has been designed requiring the pitch rate, true airspeed, angle of attack, and pitch angle. A key aspect of the design is to establish the matrix $Q$ from (42). For details, see the work of Alwi and Edwards (2008a). Principal component 
analysis based on the computed difference between the responses of the linear/nonlinear model was used to obtain $Q$ as suggested by Patton and Chen (1993).

Assume that the pitch rate, true air speed, and angle of attack $(\alpha)$ measurements are fault free and therefore

$$
F^{T}=\left[\begin{array}{llll}
0 & 0 & 0 & 1
\end{array}\right] .
$$

The reference command sequence is such that the aircraft is returned to (approximately) the initial flight conditions (Fig. 19). The nominal tracking error is shown in Fig. 20. The Root Mean Square (RMS) of the signal is 0.0150 .
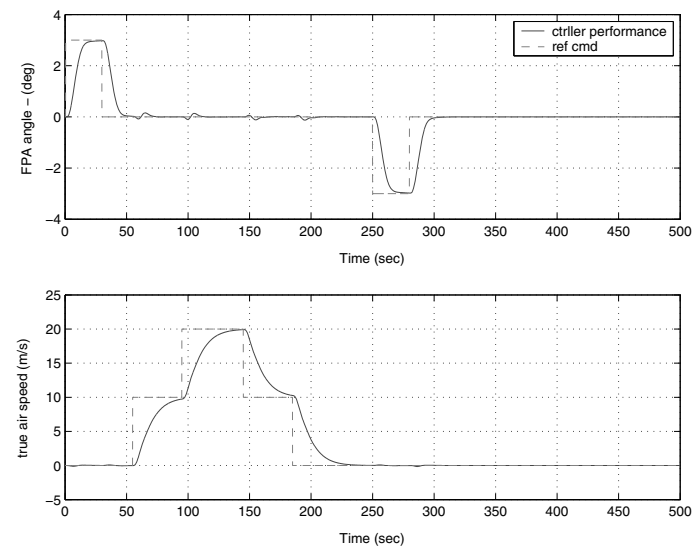

Fig. 19. Nominal closed loop performance.

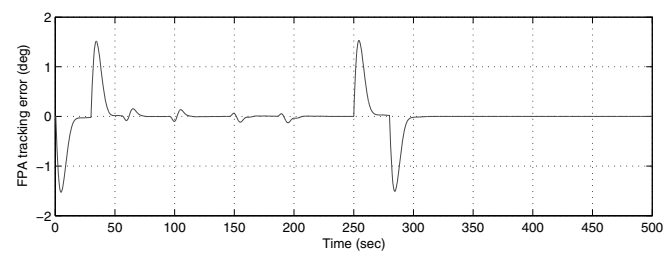

Fig. 20. Flight path tracking error.

Now a fault on the pitch sensor is introduced. This takes the form of a slowly increasing drift in the form of a ramp, which reaches a peak and then returns to nominal performance. Figure 21 shows the effect on the tracking error of using this faulty measurement in the control law. The performance of the closed loop system as shown in Fig. 21 is dangerously unacceptable. This motivates the use of the sensor fault tolerant control scheme shown in Fig. 18. The same fault as deployed in Fig. 21 is introduced. Figure 22 shows the actual fault $f_{0}$ and the estimate $\hat{f}_{0}$ obtained from the sliding mode observer. Using the sensor fault tolerant control scheme shown in Fig. 18, the closed loop performance is given in Fig. 23. Much better performance is maintained. In fact, the RMS in Fig. 23 is 0.0154 , which is close to the fault free case $(0.0150)$.

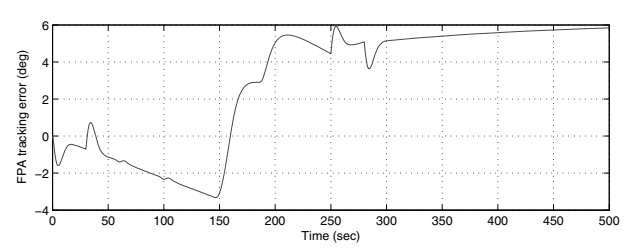

Fig. 21. Tracking error in the presence of a sensor fault (no FTC).

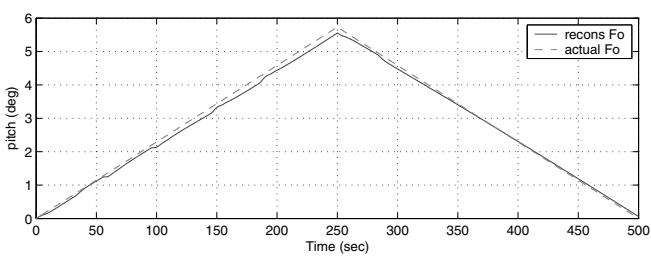

Fig. 22. Estimate of the fault signal $\hat{f}_{0}$.

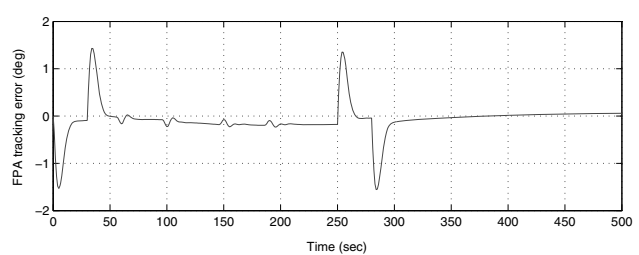

Fig. 23. Tracking error in the presence of a sensor fault (no FTC)

\section{EL-AL Bijlmermeer accident}

Figure 24 summarizes the results of piloted tests based on the EL-AL 1862 failure scenario in which engines nos. 3 and 4 detached from the right wing of a B747 and caused a significant damage (Edwards et al., 2010). The figure shows the comparisons between the implemented sliding mode CA scheme and the piloted classical controller. Figure 24 clearly shows that the proposed scheme manages to maintain nominal performance and achieve safe landing. Meanwhile, the piloted classical controller crashes during the final stage of the test flight before lining up with the runway.

\section{Conclusions}

Sliding mode methods have been historically studied because of their strong robustness properties to a certain class of uncertainty. This paper has considered how these ideas can be exploited for fault detection (specifically fault signal estimation) using sliding mode observers, and subsequently fault tolerant control. It has discussed applications of these ideas to aerospace systems. In particular, 


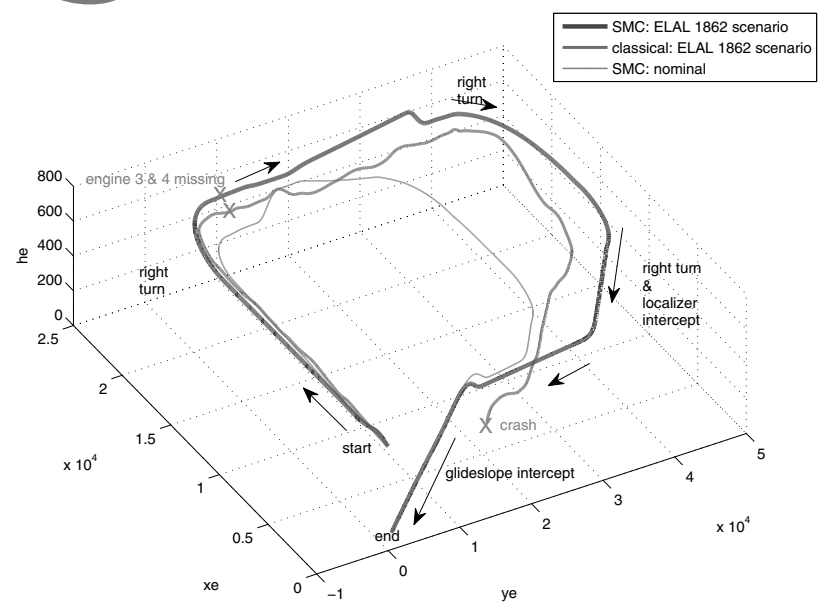

Fig. 24. EL-AL incident flight path.

piloted flight simulator results associated with the EL-AL 1862 Bijlmermeer scenario studied as part of the GARTEUR AG16 action group on fault tolerant control. The results demonstrate a successful real-time implementation of the proposed fault tolerant control scheme on a motion flight simulator configured to represent the EL-AL aircraft.

\section{Acknowledgment}

The authors gratefully acknowledge the contribution of Prof. J.A. (Bob) Mulder and Olaf Stroosma from the Aerospace Research Group at the Technical University of Delft for their work on implementing the controllers on the SIMONA simulator within the GARTEUR FM-AG16 Action Group on Fault Tolerant Control. The contribution of the other members of FM-AG16 in the discussion and development of the benchmark is also acknowledged.

\section{References}

Alwi, H. and Edwards, C. (2008a). Fault detection and faulttolerant control of a civil aircraft using a sliding-modebased scheme, IEEE Transactions on Control Systems Technology 16(3): 499-510.

Alwi, H. and Edwards, C. (2008b). Fault tolerant control using sliding modes with on-line control allocation, Automatica 44(7): 1859-1866.

Alwi, H., Edwards, C., Stroosma, O. and Mulder, J.A. (2008). Fault tolerant sliding mode control design with piloted simulator evaluation, AIAA Journal of Guidance, Control and Dynamics 31(5): 1186-1201.

Alwi, H., Edwards, C., Stroosma, O. and Mulder, J.A. (2010). Evaluation of a sliding mode fault tolerant controller for the EL-AL incident, AIAA Journal of Guidance, Control and Dynamics 33(3): 667-677.
Alwi, H., Edwards, C. and Tan, C. (2009a). Sliding mode estimation schemes for incipient sensor faults, Automatica 45(7): 1679-1685

Banda, S. (1999). Special issue editorial, International Journal of Robust and Nonlinear Control 9(14): 997-998.

Bejarano, F., Fridman, L. and Poznyak, A. (2007). Hierarchical observer for strongly detectable systems via second order sliding mode, Proceedings of the IEEE CDC'07, New Orleans, LA, USA, pp. 3709-3713.

Blanke, M., Kinnaert, M., Lunze, J. and Staroswiecki, M. (2006). Diagnosis and Fault-Tolerant Control, 2nd Edn., Springer, Berlin/Heidelberg.

Bošković, J.D. and Mehra, R.K. (2002). Control allocation in overactuated aircraft under position and rate limiting, Proceedings of the American Control Conference, Anchorage, AL, USA, pp. 791-796.

Boyd, S., Ghaoui, L.E., Feron, E. and Balakrishnan, V. (1994). Linear Matrix Inequalities in Systems and Control Theory, SIAM, Philadelphia, PA.

Buffington, J., Chandler, P. and Pachter, M. (1999). On-line system identification for aircraft with distributed control effectors, International Journal of Robust and Nonlinear Control 9(14): 1033-1049.

Chen, J. and Patton, R.J. (1999). Robust Model-Based Fault Diagnosis for Dynamic Systems, Kluwer Academic Publishers, Boston, MA.

Chen, J., Patton, R. and Zhang, H. (1996). Design of unknown input observers and robust fault detection filters, International Journal of Control 63(1): 85-105.

Chen, J. and Zhang, H. (1991). Robust detection of faulty actuators via unknown input observers, International Journal of Systems Science 22(10): 1829-1839.

Chen, W. and Saif, M. (2007). Actuator fault diagnosis for uncertain linear systems using a high-order sliding-mode robust differentiator, International Journal of Robust and Nonlinear Control 18(4-5): 413-426.

Darouach, M. (1994). On the novel approach to the design of unknown input observers, IEEE Transactions on Automatic Control 39(3): 698-699.

Davidson, J.B., Lallman, F.J. and Bundick, W.T. (2001). Realtime adaptive control allocation applied to a high performance aircraft, 5th SIAM Conference on Control \& Its Application, San Diego, CA, USA, pp. 1-11.

Dávila, A., Moreno, J.A. and Fridman, L. (2010). Variable gains super-twisting algorithm: A Lyapunov based design, IEEE American Control Conference, Baltimore, MD, USA, pp. 968-973.

Draženović, B. (1969). The invariance conditions in variable structure systems, Automatica 5(3): 287-295.

Edelmayer, A., Bokor, J., Szabó, Z. and Szigeti, F. (2004). Input reconstruction by means of system inversion: A geometric approach to fault detection and isolation in nonlinear systems, International Journal of Applied Mathematics and Computer Science 14(2): 189-199. 
Edwards, C., Lombaerts, T. and Smaili, H. (Eds.) (2010). Fault Tolerant Flight Control: A Benchmark Challenge, Lecture Notes in Control and Information Sciences, Vol. 399, Springer-Verlag, Berlin/Heidelberg.

Edwards, C. and Spurgeon, S. (1994). On the development of discontinuous observers, International Journal of Control 59(4): 1211-1229.

Edwards, C. and Spurgeon, S.K. (1998). Sliding Mode Control: Theory and Applications, Taylor \& Francis, London.

Edwards, C. and Spurgeon, S.K. (2000). A sliding mode control observer based FDI scheme for the ship benchmark, European Journal of Control 6(4): 341-356.

Edwards, C., Spurgeon, S. and Patton, R. (2000). Sliding mode observers for fault detection, Automatica 36(4): 541-553.

Edwards, C. and Tan, C.P. (2006). A comparison of sliding mode and unknown input observers for fault reconstruction, $E u$ ropean Journal of Control 12(3): 245-260.

Enns, D. (1998). Control allocation approaches, AIAA Guidance, Navigation and Control Conference and Exhibit, Boston, MA, USA, pp. 98-108.

Floquet, T., Edwards, C. and Spurgeon, S. (2007). On sliding mode observers for systems with unknown inputs, International Journal of Adaptive Control and Signal Processing 21(8-9): 638-656.

Forssell, L. and Nilsson, U. (2005). ADMIRE, the aero-data model in a research environment version 4.0: Model description, Technical Report FOI-R-1624-SE, Swedish Defence Agency (FOI), Stockholm.

Fridman, L., Davila, J. and Levant, A. (2007). High-order sliding-mode observation and fault detection, Proceedings of the IEEE Conference on Decision and Control, New Orleans, LA, pp. 4317-4322.

Härkegård, O. and Glad, S.T. (2005). Resolving actuator redundancy-Optimal control vs. control allocation, Automatica 41(1): 137-144.

Haskara, I., Ozguner, U. and Utkin, V. (1998). On sliding mode observers via equivalent control approach, International Journal of Control 71(6): 1051-1067.

Hess, R.A. and Wells, S.R. (2003). Sliding mode control applied to reconfigurable flight control design, Journal of Guidance, Control and Dynamics 26(3): 452-462.

Krasnova, S., Utkin, V. and Mikheev, Y. (2001). Cascade design of state observers, Automation and Remote Control 62(2): 207-226.

Levant, A. (2003). Higher-order sliding modes, differentiation and output-feedback control, International Journal of Control 76(9-10): 924-41.

Moreno, J.A. and Osorio, M. (2008). A Lyapunov approach to second-order sliding mode controllers and observers, $47 \mathrm{th}$ IEEE Conference on Decision and Control, Cancun, Mexico, pp. 2856-2861.

Ng, K., Tan, C., Edwards, C. and Kuang, Y. (2007). New results in robust actuator fault reconstruction in linear uncertain systems, International Journal Robust and Nonlinear Control 17(4): 1294-1319.
Patton, R. (1997). Robustness in model-based fault diagnosis: The 1997 situation, IFAC Annual Reviews 21: 101-121.

Patton, R. and Chen, J. (1993). Optimal unknown input distribution matrix selection in robust fault diagnosis, Automatica 29(4): 837-841.

Patton, R., Frank, P. and Clark, R. (1989). Fault Diagnosis in Dynamic Systems: Theory and Application, Prentice Hall, New York, NY.

Saif, M. and Guan, Y. (1993). A new approach to robust fault detection and identification, IEEE Transactions on Aerospace and Electronic Systems 29(3): 685-695.

Sharam, R. and Aldeen, M. (2007). Fault detection in nonlinear systems with unknown inputs using sliding mode observer, Proceedings of the American Control Conference, New York, NY, USA, pp. 432-437.

Shtessel, Y., Buffington, J. and Banda, S. (2002). Tailless aircraft flight control using multiple time scale re-configurable sliding modes, IEEE Transactions on Control Systems Technology 10(2): 288-296.

Tan, C. and Edwards, C. (2010). Robust fault reconstruction in uncertain linear systems using multiple sliding mode observers in cascade, IEEE Transactions on Automatic Control 55(4): 855-867.

Tan, C.P. and Edwards, C. (2002). Sliding mode observers for detection and reconstruction of sensor faults, Automatica 38(2): 1815-1821.

Tan, C.P. and Edwards, C. (2003). Sliding mode observers for robust detection and reconstruction of actuator and sensor faults, International Journal of Robust and Nonlinear Control 13(5): 443-463.

Theilliol, D., Join, C. and Zhang, Y. (2008). Actuator fault tolerant control design based on a reconfigurable reference input, International Journal of Applied Mathematics and Computer Science 18(4): 553-560, DOI: 10.2478/v10006008-0048-1.

Utkin, V.I. (1992). Sliding Modes in Control Optimization, Springer-Verlag, Berlin.

Wang, J., Tsang, K., Li, G. and Zhang, L. (2003). Cascade observer-based fault diagnosis for nonlinear systems, Proceedings of the IASTED International Conference on Modelling, Simulation and Optimization, Banff, Alberta, Canada, pp. 253-258.

Zhou, K., Doyle, J. and Glover, K. (1996). Robust and Optimal Control, Prentice Hall, Upper Siddle River, NJ.

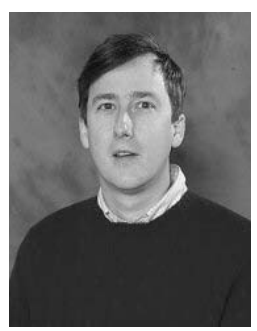

Christopher Edwards was born in Swansea, South Wales. He graduated from Warwick University in 1987 with a B.Sc. in mathematics. In 1987-91 he was employed as a research officer for British Steel Technical in Port Talbot, where he was involved with mathematical modelling of rolling and finishing processes. In 1991 he moved to Leicester University as a Ph.D. student supported by a British Gas Research Scholarship and was awarded a Ph.D. in 1995. He was appointed as a lecturer in the Control Systems Research Group at Leicester University in 1996, and promoted to a senior lecturer in 2004 and a reader in 2007 . He is a co-author of over 180 refereed papers and two books on sliding mode control. 


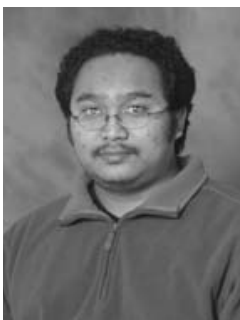

Halim Alwi was born in Klang, Malaysia. After completing his secondary school education at the Royal Military College, Kuala Lumpur, Malaysia, he studied at the University of Leicester, UK, and graduated in 2000 with a B.Eng. (Hons.) degree in mechanical engineering. In 2000-2004 he was an engineer at the New Straits Times Press, Malaysia, where he was involved in the installation and commissioning of a new automated system for the mailroom, as well as overseeing the maintenance of mailroom machines. He obtained his Ph.D. in 2008 from the University of Leicester in the area of fault tolerant control applied to aerospace systems.

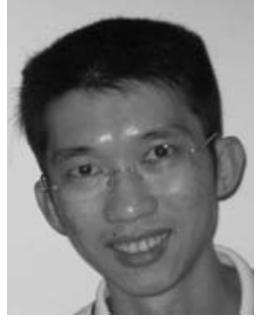

Chee Pin Tan obtained a B.Eng. (Hons.) in electrical and electronic engineering and a Ph.D. from the University of Leicester, UK, in 1998 and 2002, respectively. Currently he is a senior lecturer at the School of Engineering, Monash University (Sunway Campus) in Malaysia. His research interests include sliding mode observers and robust fault reconstruction.

Received: 23 January 2011

Revised: 23 June 2011 